Published on Web 05/02/2007

\title{
Charge Injection Across Self-Assembly Monolayers in Organic Field-Effect Transistors: Odd-Even Effects
}

\author{
Pablo Stoliar, ${ }^{\dagger}$ Rajendra Kshirsagar,${ }^{\dagger}$ Massimiliano Massi, ${ }^{\dagger}$ Paolo Annibale,${ }^{\dagger}$ \\ Cristiano Albonetti, ${ }^{\dagger}$ Dago M. de Leeuw, ${ }^{\S}$ and Fabio Biscarini ${ }^{*}, \dagger$ \\ Contribution from the Nanotechnology of Multifunctional Materials (NMM) Research Division, \\ CNR-ISMN, Via Gobetti 101, I-40129 Bologna, Italy, and Philips Research Laboratories, \\ High Tech Campus 4, NL-5656 AA, Eindhoven, The Netherlands \\ Received December 22, 2006; E-mail: f.biscarini@bo.ismn.cnr.it
}

\begin{abstract}
We investigate the role of self-assembly monolayers in modulating the response of organic fieldeffect transistors. Alkanethiol monolayers of chain length $n$ are self-assembled on the source and drain electrodes of pentacene field-effect transistors. The charge carrier mobility $\mu$ exhibits large fluctuations correlated with odd-even $n$. For $n<8, \mu$ increases by 1 order of magnitude owing to the decrease of the hole injection barrier and the improved molecular order at the organic-metallic interface. For $\mathrm{n} \geq 8, \mu$ decays exponentially with an inverse decay length $\beta=0.6 \AA^{-1}$. Our results show that (i) charge injection across the interface occurs by through-bond tunneling of holes mediated by the alkanethiol layer; (ii) in the long-chain regime, the charge injection across the alkanethiol monolayer completely governs the transistor response; (iii) the transistor is a sensitive gauge for probing charge transport across single monolayers. The odd-even effect is ascribed to the anisotropic coupling between the alkanethiol terminal $\sigma$ bond and the HOMO level of ordered pentacene molecules.
\end{abstract}

\section{Introduction}

Alkanethiol self-assembly monolayers $\left(\mathrm{SAMs}^{1}\right)$ are widely investigated as prototype systems for molecular electronics. ${ }^{2}$ Charge transport across alkanethiol SAMs has been investigated in a variety of $\mathrm{Au} / \mathrm{molecule/metal}$ junctions, where the metal counterelectrode is made with either vacuum-sublimed $\mathrm{Au}$ films, ${ }^{3}$ conductive scanning probes, ${ }^{4}$ mercury droplets, ${ }^{5,6}$ or by mechanically controlled break junctions. ${ }^{7}$ A scalable device architecture based on alkanedithiols and a polymer conductor as counter-electrode ${ }^{8}$ has demonstrated that SAMs can be used as active components in molecular electronics devices. In all these experiments, the conductance of the monolayer junction decays exponentially versus the chain length $n$ (expressed as the number of $\mathrm{C}$ atoms in the alkyl chain, or methylene units) as $\exp (-\beta n)$. The measured inverse decay rate $\beta$ lies always in the range $0.5-1 \AA^{-1}$, which strongly suggests that charge transport takes place via through-bond tunneling assisted by the alkanethiol $\sigma$ bonds. Whether electrons or holes are transported through the junction has not been conclusively established

\footnotetext{
CNR-ISMN

§ Philips Research Laboratories.

(1) Ulman, A. Chem. Rev. 1996, 96, 1533-1554.

(2) Salomon, A.; Cahen, D.; Lindsay, S.; Tomfohr, J.; Engelkes, V. B.; Frisbie, C. D. Adv. Mater. 2003, 15, 1881-1890.

(3) Wang, W; Lee, T.; Reed, M. A. Phys Rev B 2003, 68, 035416.

(4) Wold, D. J.; Frisbie, C. D. J. Am. Chem. Soc. 2000, 122, 2970-2971.

(5) Holmlin, R. E.; Haag, R.; Chabinyc, M. L.; Ismagilov, R. F.; Cohen, A

E.; Terfort, A.; Rampi, M. A.; Whitesides, G. M. J. Am. Chem. Soc. 2001, 123, 5075-5085.

(6) Wang, W. Y.; Lee, T.; Reed, M. A. Rep. Prog. Phys. 2005, 68, 523-544.

(7) Reed, M. A.; Zhou, C.; Muller, C. J.;Burgin, T. P.; Tour, J. M. Science 1997, 278, 252-254

(8) Akkerman, H. B.; Blom, P. W. M.; de Leeuw, D. M.; de Boer, B. Nature 2006, 441,69 .
}

10.1021/ja069235m CCC: $\$ 37.00$ @ 2007 American Chemical Society because these configurations cannot discriminate the nature of charge carriers.

Conversely, in organic electronics, the functionalization of the source and drain Au contacts with alkanethiol SAMs is known to improve the effective charge mobility and the response of organic field-effect transistors (FETs). ${ }^{\text {aa }}$ The observation of a homogeneous density of organic semiconductor domains at the contact-channel interface is invoked to qualitatively explain the effect. ${ }^{\mathrm{gb}}$ Neither insight on how the SAM affects charge injection or the electronic structure of the interface nor the finer details of the molecular organization in relation to the growth conditions of the organic semiconductor have been explored or understood.

In this paper, we investigate the influence of alkanethiol monolayers on the response of a real organic FET. We modulate the charge injection interface between the Au electrodes (source and drain) and the organic semiconductor (pentacene) by inserting $n$-alkanethiols, whose chain length $n$ is systematically varied in the range between 3 and 16 units.

The charge mobility of holes in the transistor versus $n$ exhibits novel and counterintuitive features: (i) large odd-even fluctuation throughout the whole range of $n$; (ii) a nonmonotonic trend versus $n$, with a maximum for $n=8$; (iii) an exponential decay at large $n$, indicating that charge transport across the alkanethiol monolayer completely controls the response of the device. We analyze the correlation between the behavior of the charge

(9) (a) Kymissis, I.; Dimitrakopoulos, C. D.; Purushothaman, S. IEEE Trans. Electron Devices 2001, 48, 1060-1064. (b) Dimitrakopoulos, C. D.; Malenfant, P. R. L. Adv. Mater. 2002, 14, 99-117. 
mobility, the electronic structure of the injection interface, ${ }^{10,11}$ and the morphology of the semiconductor layer at the injection interface. The odd-even effect is the direct evidence of charge injection mediated by a through-bond mechanism across the alkanthiols. Time-dependent perturbation theory indicates that the odd-even effects can be associated to the anisotropic coupling between the last $\sigma$ bond in the alkanethiol chain and the HOMO of pentacene.

Our work demonstrates the sensitivity of the organic transistors to the fine structure of the injection interface down to submolecular details. It also shows that the organic FET is a robust experimental gauge for measuring charge transport across monolayers.

This paper is organized as follows: In section II Results we show a direct evidence by electrostatic force microscopy (EFM) of the effect of the SAM on the organic/metal interface of the device which explains the essential features of our experiment. Then we present the experimental data from the device characteristics and morphology analysis from atomic force microscopy (AFM). In section III Discussion, we relate the device model to the electronic structure of the interface, and explain the odd-even effect with time-dependent perturbation theory. In the Conclusions, we summarize our results. The Supporting Information (SI) contains the experimental section, electrical characterization data, and supplementary AFM and EFM data.

\section{Results}

II.1. Device Model and Electrostatic Potential Profile. In Figure 1a we show a schematic drawing of the device configuration used in the experiment. In an organic FET, charge carriers are generated as the gate voltage $V_{\mathrm{gs}}$ is turned on. When the device is operated in the saturation regime, namely, $\left|V_{\mathrm{ds}}\right|>$ $\left|V_{\mathrm{gs}}-V_{\mathrm{T}}\right|$, charge carriers move within a narrow region of the organic semiconductor, whose physical size corresponds to a few molecular layers, in the proximity of the gate dielectric layer. ${ }^{12}$ Upon the application of a bias voltage $V_{\mathrm{ds}}$ to the electrodes, a current flows across the channel between the source and drain electrodes. Continuity in the current implies that charge carriers are injected across the interface between the organic semiconductor and the source and drain electrodes.

The electrostatic potential in a real transistor does not vary linearly between source and drain owing to the interface resistance. ${ }^{13,14}$ This can be directly imaged by EFM (see SI for details). Figure $1 \mathrm{~b}$ shows the electrostatic potential profiles measured across the channel of real organic FETs during operations. The electrostatic potential undergoes a rapid drop near the source contact and a smaller jump near the drain electrode. Thus, the longitudinal field in the channel is smaller than the nominal electric field $V_{\text {ds }}$ divided by the channel length $L$. The effective gate field, proportional to $V_{\mathrm{gs}}-V_{\mathrm{rcs}}$, inside the semiconductor layer is decreased, resulting in a smaller

(10) Conjugated Polymer and Molecular Inerfaces: Science and Technology for Photonic and Optoelectronic Applications, Salaneck, W. R., Seki, K. Kahn, A., Pireaux, J.-J., Eds.; Marcel Dekker: New York, 2001.

(11) Kahn, A.; Koch, N.; Gao, W. J. Polym. Sci., Part B: Polym. Phys. 2003 41, 2529-2548.

(12) Dinelli, F.; Murgia, M.; Levy, P.; Cavallini, M.; Biscarini, F.; de Leeuw, D. M. Phys. Rev. Lett. 2004, 92, 116802.

(13) Bürgi, L.; Sirringhaus, H.; Friend, R. H. Appl. Phys. Lett. 2002, 80, $2913-$ 2915.

(14) Puntambekar, K. P.; Pesavento, P. V.; Frisbie, C. D. Appl. Phys. Lett. 2003, $83,5539-5541$

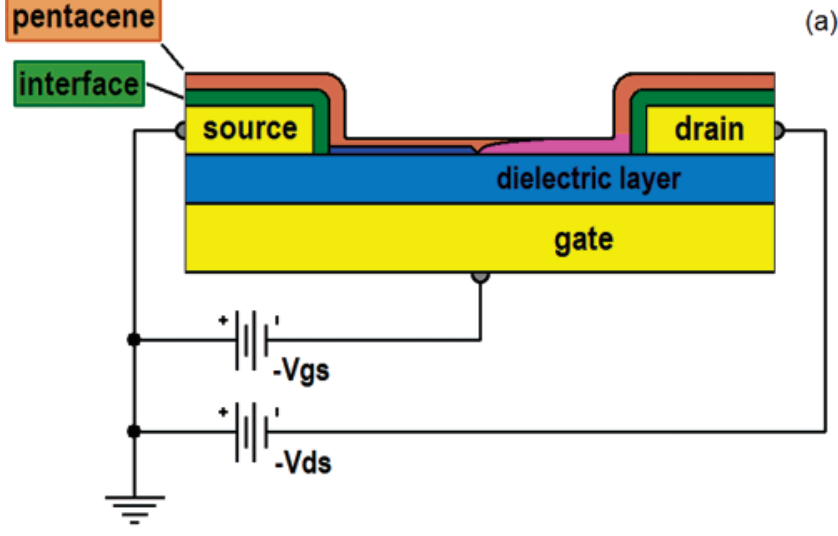

(b)

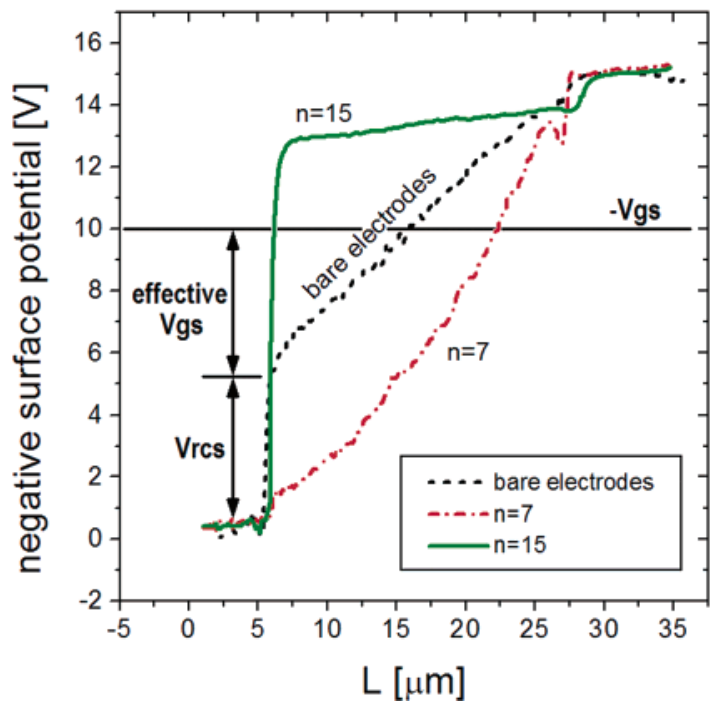

Figure 1. (a) Model for the organic field-effect transistor working in the saturation regime, viz. $\left|V_{\mathrm{ds}}\right|>\left|V_{\mathrm{gs}}-V_{\mathrm{T}}\right|$. A p-type semiconductor is shown, viz. charge carriers are holes. Charge carriers are formed in the proximity of the dielectric layer (blue region) as the gate voltage $V_{\mathrm{gs}}$ is turned on. When the transistor operates in the saturation regime, a region depleted of charge carriers is formed (purple region). (b) Profiles of the electrostatic potential across the device channel measured by electrostatic force microscopy (EFM) for $n=0$ (black dotted line), $n=7$ (red dot-dashed line), and $n=15$ (green continuous line). The voltage at the source contact $V_{\text {rcs }}$, the effective $V_{\mathrm{gs}}$, that is the difference between $V_{\mathrm{gs}}$ and the local electrostatic potential, and the extent of the depletion region are shown for the device with bare electrodes (black line).

number of charge carriers. ${ }^{15}$ The maximum current that is forced through the channel is lower than expected. Since the effective charge mobility $\mu$ scales as the maximum current, it will also decrease.

The different profiles in Figure 1b show that the potential profile of the device is extremely sensitive to the chemical functionalization of the interface. The insertion of short-chain alkanethiol (red line) reduces the voltage drop at the interface with respect to the device with bare Au electrodes. This leads to an increased saturation current. For the long-chain alkanethiol $n=15$ (green line) the large potential drop at the contacts yields an almost flat potential in the channel. Maximum current will be limited in this case by the total SAM resistance.

By comparing the three cases, we infer that the behavior of the interfacial resistance versus $n$ will not exhibit a monotonic

(15) (a) Bürgi, L.; Richards, T. J.; Friend, R. H.; Sirringhaus, H. J. Appl. Phys 2003, 94, 6129-6137. (b) Natali, D.; Fumagalli, L.; Sampietro, M. J. Appl. Phys. 2007,101, 014501. 

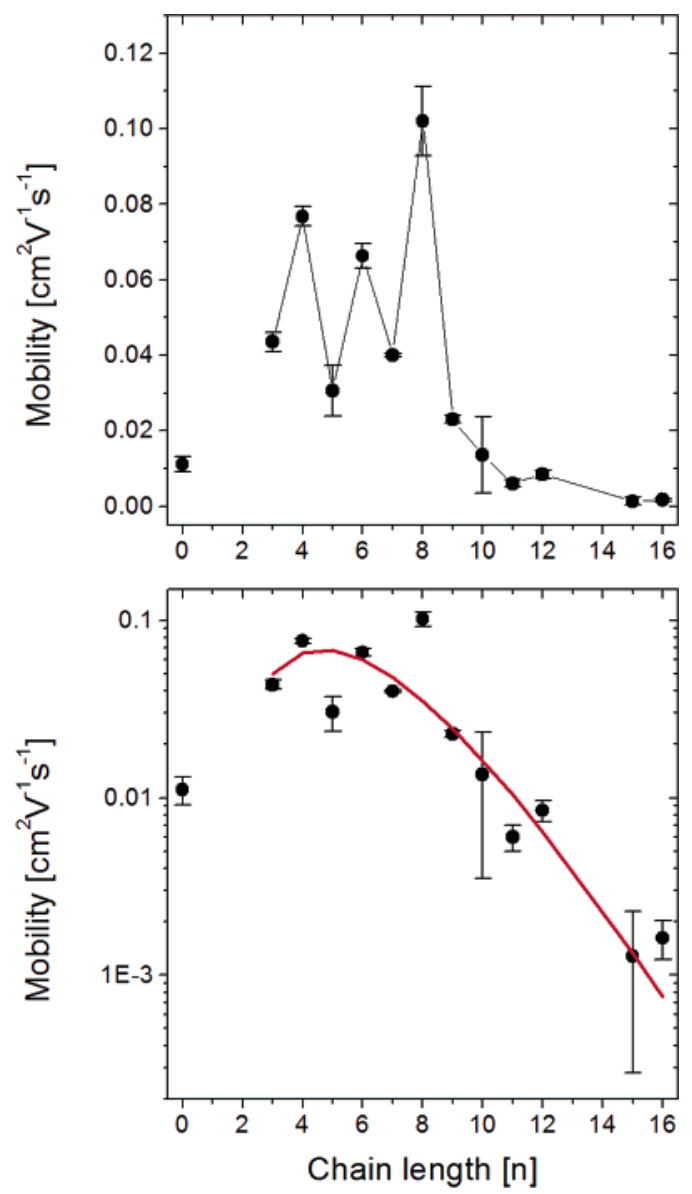

Figure 2. (a) Charge mobility of pentacene FETs vs alkanethiol chain length $n$ as obtained in the saturation regime. (b) The same data as in (a) in a semilog plot. The geometrical mean of the fitting functions of the odd and even sequence $\bar{\mu}(n)=\mu_{1} n^{\left(\alpha_{\text {odd }}+\alpha_{\text {even }}\right) / 2} \exp \left[-1 / 2\left(\beta_{\text {odd }}+\beta_{\text {even }}\right)\right] n=\mu_{1} n^{3.75}$ $\exp (-0.81 n)$ highlights the odd-even effect and the exponential decay at large chain length (solid line).

trend but it would go through a minimum at some intermediate chain length. The consequence of the interface functionalization on the effective charge mobility, which is extracted from the transfer characteristics of the device, as detailed in the SI section, is presented in the next paragraph.

II.2. Organic FET Response. Figure 2 shows the evolution of the charge mobility $\mu$ versus $n$ in the organic FET. The charge mobility increases by 1 order of magnitude as $n$ increases from zero to eight, reaches a maximum, then decays exponentially at large $n$. The fluctuations of the mobility values with $n$ exhibit a clear odd-even effect of the chain length on the charge mobility, with higher mobility for even- $n$ terms.

The global fit of the data yields a large $\chi$-square coefficient owing to the odd-even fluctuations. We then fit the odd and even series separately using the function

$$
\mu(n)=\mu_{1} n^{\alpha} \mathrm{e}^{-\beta n}
$$

The choice of this function is explained in the Discussion section. The power law increase at short chain length exhibits an exponent $\alpha=3.3 \pm 1.3$ (even terms) and $4.2 \pm 0.9$ (odd terms), their mean value being $\alpha=3.8 \pm 1.1$. The fast decay of the charge mobility at large $n$ indicates that in this range of $n$ the charge injection controls the device response. For $n>8$ the exponential decay rate $\beta$ is $0.90 \pm 0.19$ (odd) and $0.72 \pm$

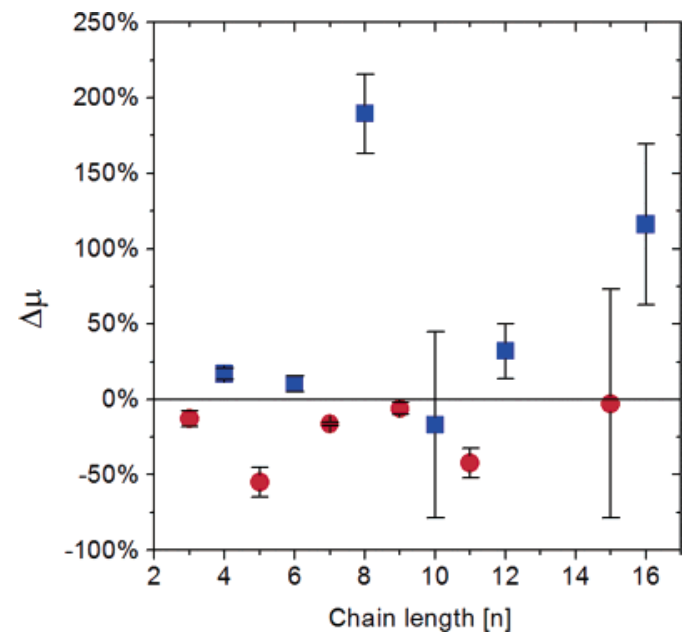

Figure 3. Relative odd-even fluctuations (scaled to the mean mobility $\bar{\mu}(n))$. In average, the even terms (blue) are raised $17.8 \%$ and the odd terms (red) are lowered $16.2 \%$.

0.20 (even) inverse methylene units $\left(\mathrm{CH}_{2}{ }^{-1}\right)$. Their average value $\beta=0.81 \pm 0.20 \mathrm{CH}_{2}{ }^{-1}$ translates into $\beta=0.54( \pm 0.13)-$ $0.62( \pm 0.15) \AA^{-1}$ depending on the structural data chosen for the alkanethiol layer. ${ }^{16,17}$ Both the mean and the odd/even values of $\beta$ are in the range of the values reported for the current across single alkanethiol molecule junctions $\left(0.5-1 \AA^{-1}\right) .^{2}$ Our result is also in remarkable agreement with the decay rate $\beta=0.57-$ $0.66 \AA^{-1}$ reported for single monolayer rectifiers based on alkanedithiol SAMs. ${ }^{8}$ Noticeably, no earlier charge transport experiments across single molecules reported odd-even effects in the current versus $n$ trend.

The odd-even fluctuations, although seeming to damp out with increasing $n$, remain of comparable relative intensity throughout the range. This is shown in Figure 3 where the relative fluctuation $\Delta \mu=\mu(n) / \bar{\mu}(n)-1$ of the charge mobility with respect to the mean value $\bar{\mu}(n)$ (as defined in the caption of Figure 2) allows us to compare data in a range of currents spanning several orders of magnitude.

The spread of $\Delta \mu$ is $\Delta \mu_{\text {even }}=(17.8 \pm 3) \%$ for the even terms and $\Delta \mu_{\text {odd }}=(-16.2 \pm 1) \%$ for the odd ones. The negative value for the odd terms indicates that they are always under the even terms.

We have also measured the effective resistance of the channel $R=V_{\mathrm{ds}} / I_{\mathrm{max}}$ versus $n$ in the linear (low-voltage) regime (Figure $1, \mathrm{SI}) . R$ increases rapidly (exponential fit yields a constant of 0.26 inverse methylene units) without exhibiting any clear oddeven effect. ${ }^{17}$ Albeit the scattering of data does not allow us to unambiguously rule out the presence of odd-even effect, these data suggest that in this regime the alkanethiol layer acts as an insulating geometrical barrier, and it is therefore less sensitive to the electronic structure of the interface.

II.3. Analysis of the Pentacene Film Morphology. To understand the relevance of the morphology in the observed trend of mobility versus chain length, we studied the evolution of the interface morphology by AFM. Upon the chosen growth conditions, pentacene molecules on hexamethyldisilazane

(16) Porter, M. D.; Bright, T. B.; Allara, D. L.; Chidsey, C. E. D. J. Am. Chem. Soc. 1987, 109, 3559-3568.

(17) Engelkes, V. B.; Beebe, J. M.; Frisbie, C. D. J. Am. Chem. Soc. 2004 126, 14287-14296.

(18) Verlaak, S.; Steudel, S.; Heremans, P.; Janssen, D.; Deleuze, M. S. Phys. Rev. B: Condens. Matter Mater. Phys. 2003, 68, 195409. 

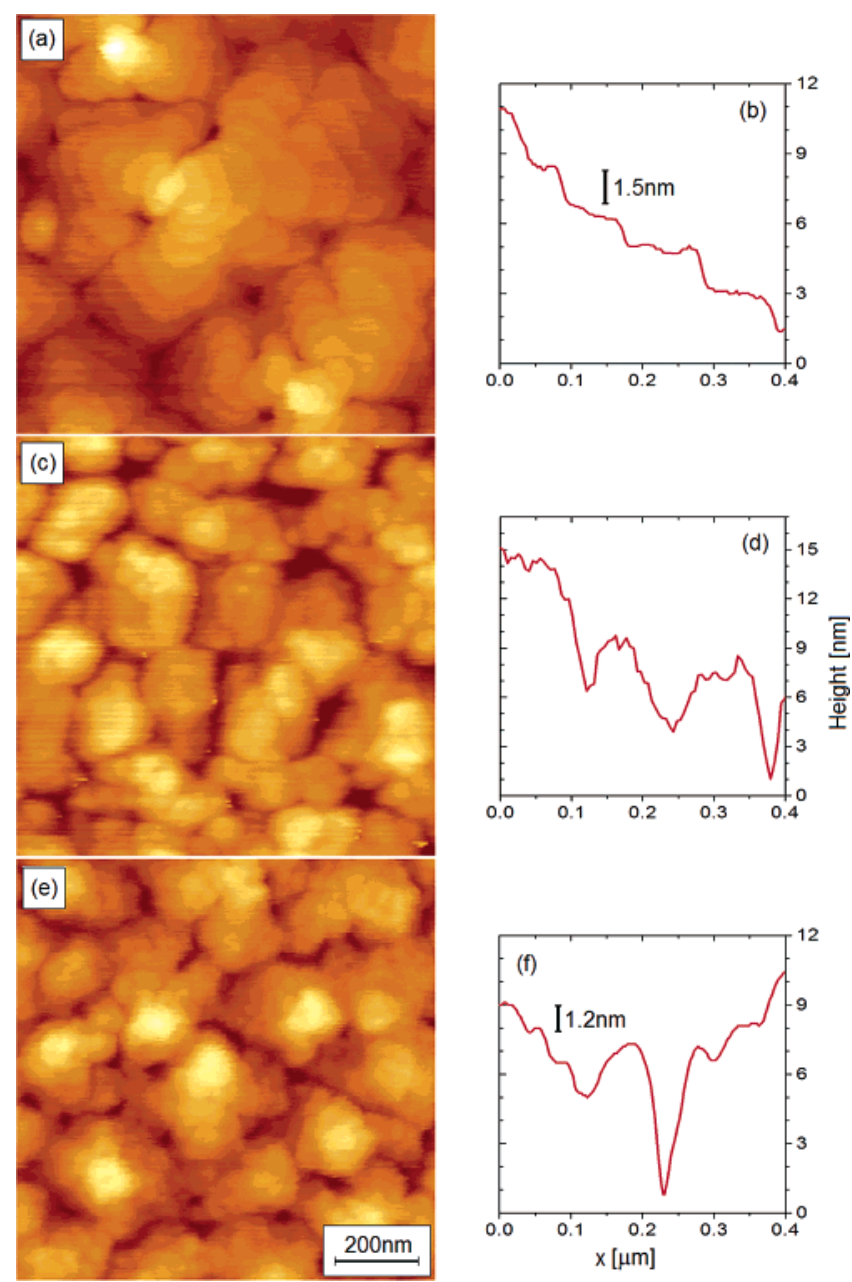

Figure 4. Morphology of pentacene thin film (10 nm thickness) in FETs: (a) layered islands with terraces on HMDS/SiOx; (b) line profile across panel a exhibits $1.5 \pm 0.1 \mathrm{~nm}$ terrace height; (c) compact islands on bare gold electrodes; (d) line profile across panel c; (e) layered islands on octanethiol functionalized electrodes; (f) line profile across panel e exhibits $1.2 \pm 0.1 \mathrm{~nm}$ terrace height.

(HMDS)-primed silicon oxide form layered islands ${ }^{18}$ as shown in Figure 4. This morphology is consistent with that reported in pentacene growth studies on dielectric surfaces ${ }^{19,20}$ and on transistors. $^{21}$

Each layer is $1.5 \pm 0.1 \mathrm{~nm}$ high (Figure $4 \mathrm{~b}$ ), indicating that pentacene molecules are stacked with their long axes normal to the surface, consistently with the $c$-lattice distance in the thinfilm phase $(1.56 \mathrm{~nm}){ }^{19}$ Conversely, the morphology of the pentacene film grown on the bare gold electrode (Figure 4c) exhibits round shaped grains with no evidence of molecular terraces (Figure 4d). Molecular layers appear clearly on the islands grown on electrodes functionalized with long-chain alkanethiols as in Figure 4e. In this case, the height of the terraces is an integer multiple of $1.2 \pm 0.1 \mathrm{~nm}$ (Figure $4 \mathrm{f}$ ), close to the $1.45 \mathrm{~nm}$ of the bulk phase. ${ }^{22}$ This evidence indicates that

(19) Mayer, A. C.; Ruiz, R.; Zhou, H.; Headrick, R. L.; Kazimirov, A.; Malliaras, G. Phys. Rev. B: Condens. Matter Mater. Phys. 2006, 73, 205307.

(20) Pratontep, S.; Nuesch, F.; Zuppiroli, L.; Brinkmann, M. Phys. Rev. B: Condens. Matter Mater. Phys. 2005, 72, 085211.

(21) Ruiz, R.; Papadimitratos, A.; Mayer, A. C.; Malliaras, G. G. Adv. Mater. 2005, 17, 1795-1798.

(22) Campbell, R. B.; Robertson, J. M.; Trotter, J. Acta Crystallogr. 1961, 14, 705-711.
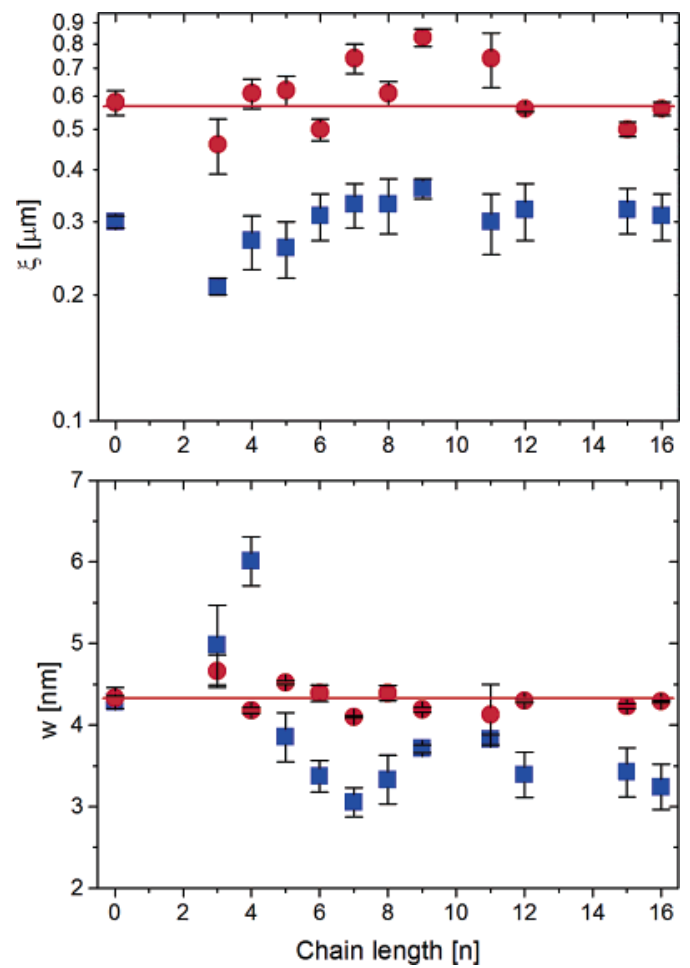

Figure 5. (a) Correlation length $\xi$ and (b) saturated roughness $w$ vs chain length $n$ for pentacene thin films on electrodes (squares) and channel (circles).

alkanethiols promote a molecularly ordered pentacene growth at the organic/metal interface.

The effect on the morphology of the growing pentacene layer is rather subtle and cannot be easily inferred from the visual inspection of AFM images of pentacene thin films on the electrodes (see also Figure 2 in SI). We have therefore measured the morphological properties, namely, correlation length and saturation roughness, ${ }^{23}$ of the pentacene thin films grown on top of the electrodes by means of the power spectrum density analysis. ${ }^{12,23}$ The nucleation density on the electrodes decreases, and the grains grow larger in size, as $n$ increases at short chain length, then saturates at larger $n$. This is apparent from the evolution of the correlation length (Figure 5a) that represents the average grain size. This change is accompanied by progressive decrease of the pentacene roughness (Figure 5b) at short chain length followed by saturation to a plateau.

The plateau in both correlation length and roughness indicates that the morphology on the electrodes becomes invariant for $n$ $>6-8$. Noticeably, the morphological properties of pentacene films grown on HMDS-functionalized channels do not substantially change with the different alkanethiols. It is also important to mention that roughness and correlation length of our bare $\mathrm{Au}$ electrodes are 0.7 and $150 \mathrm{~nm}$, respectively, showing that pentacene does not grow on the electrodes conformally with the underlying Au film. Since the amount of pentacene in each sample was the same, the combined results in Figure 4 and Figure 5 indicate that the pentacene interface evolves toward a more compact 2D morphology as $n$ increases. Thus, the charge is injected from the electrodes into a pentacene layer that becomes more ordered both at the molecular scale and in the long-range as $n$ increases.

(23) Biscarini, F.; Samori, P.; Greco, O.; Zamboni, R. Phys. Rev. Lett. 1997, $78,2389-2392$. 

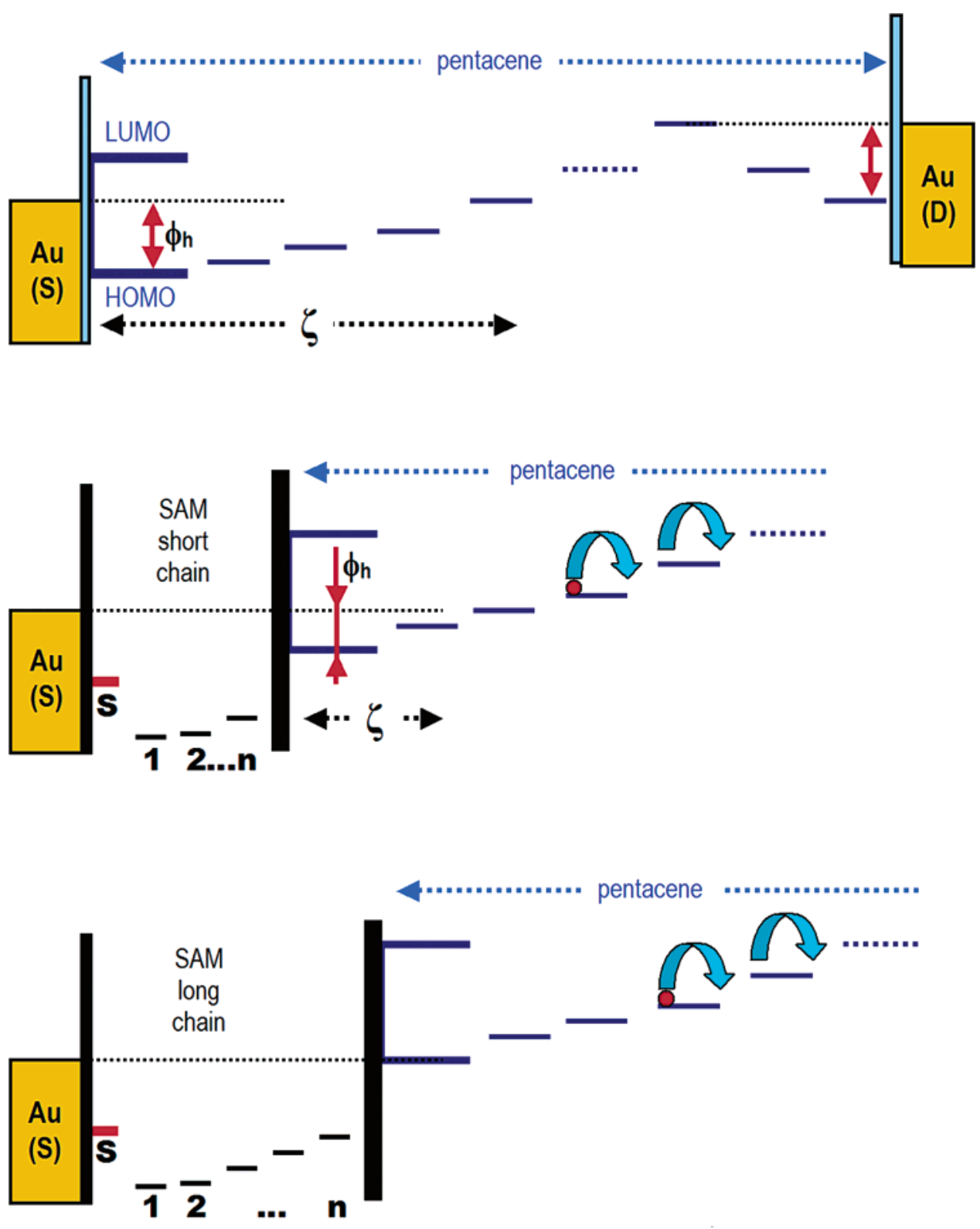

Figure 6. Schematic energy diagram of the (a) pentacene/Au interface and (b, c) pentacene/SAM/Au interface. The thickness of the HOMO states reflects the "broadening" of the HOMO band due to the hybridization with the surface density of states.

\section{Discussion}

The mobility data in Figure 2 clearly show that the alkanethiol spacer between pentacene and gold largely controls the charge mobility of the device and that the FET is extremely sensitive to subtle fluctuations of the interface. To understand the trend observed, namely, a maximum in the charge mobility, we discuss the EFM result in Figure 1 in terms of the structure of the pentacene/alkanethiol/Au junction. Thus, we introduce a length scale of charge injection.

III.1. Length Scale of Charge Injection. The electronic factors that determine the resistance of the organic/metal interface have been addressed by photoelectron spectroscopy. These studies on ideal metal/organic interfaces, ${ }^{24}$ have pointed out the role of surface dipoles and polarizability in shifting the electronic levels of the adsorbed molecules with respect to their

(24) Witte, G.; Lukas, S.; Bagus, P. S.; Wöll, C. Appl. Phys. Lett. 2005, 87, 263502 position in the unperturbed molecules, thus creating an energy barrier for injection of the charge carriers from the electrode to the organic semiconductor. ${ }^{25}$ The schematic energy diagram of the organic-metal interface in a pentacene FET is shown in Figure 6a. This picture is qualitatively consistent with the trend of the voltage drops at the interfaces reported in Figure 1b. On bare electrodes, pentacene molecules, adsorb preferentially flat on gold (111) facets, namely, the dominant exposed surface in $\mathrm{Au}$ thin films (see SI), to maximize their interaction with the surface via $\pi$-electrons. ${ }^{26,27}$ The planar adsorption generates a surface dipole that shifts down the HOMO level of pentacene by $0.85 \mathrm{eV}$ below the Au Fermi level (5.2 eV work function in vacuum), ${ }^{11}$ introducing an additional barrier $\Phi_{\mathrm{h}}$ to hole injection (Figure 6a). The unperturbed pentacene HOMO $(-5.1 \mathrm{eV})$ is

(25) Amy, F.; Chan, C.; Kahn, A. Org. Electron. 2005, 6, 85-91.

(26) Hu, W. S.; Tao, Y. T.; Hsu, Y. J.; Wei, D. H.; Wu, Y. S. Langmuir 2005, $21,2260-2266$

(27) Corradini, V.; Menozzi, C.; Cavallini, M.; Biscarini, F.; Betti, M. G.; Mariani, C. Surf. Sci. 2003, 532-535, 249-254. 
almost in resonance with the Au Fermi level. ${ }^{11}$ The HOMO levels of pentacene molecule in the channel undergo a linear shift because of the effective longitudinal field.

Planar adsorption of pentacene molecules induces threedimensional nucleation. This is consistent with recent reports by Witte et al. ${ }^{28,29}$ This disordered nucleation is amplified at larger length scales as pentacene film grows into grains, as observed in Figure 4b. There is therefore a mismatch in the energy levels and molecular disorder with respect to the pentacene molecules in the channel, which nucleate and grow normal to the dielectric surface. ${ }^{19,30,31}$ The consequence of this different nucleation and growth on the electrodes with respect to the channel is that a region of interfacial disorder, both in energy and the molecular organization, is formed in the proximity of the electrodes. ${ }^{29}$ The length scale $\zeta$ of interfacial disorder exceeds the molecular scale of the pentacene layer adsorbed on Au. Charge carriers have to cross the disordered regions at the electrodes to have a current through the device channel.

The insertion of alkanethiols between pentacene and gold introduces three competing effects: (i) the thickness of the "geometrical" barrier between pentacene and Au increases with $n,{ }^{2}$ (ii) the hole injection barrier of pentacene decreases versus $n$; (iii) the length scale of interfacial disorder $\zeta$ decreases with $n$. We coarsegrain these effects by introducing the lengthscale of charge injection $\lambda$ as

$$
\lambda=\text { constant }+(n-1) l+\zeta(\mathrm{n})
$$

where the first two terms on the right side add to the thickness of the alkanethiol, being the constant equal to the sum of the equilibrium distance $r_{\mathrm{Au}-\mathrm{S}}$ between $\mathrm{Au}$ and $\mathrm{S}$ in the thiolate, the $\mathrm{S}-\mathrm{C}$ bond length $r_{\mathrm{S}-\mathrm{C}}$, and the equilibrium distance of pentacene on the alkanethiol layer, $r_{\mathrm{Pen}}$. With $l$ we indicate the bond length between two methylene units. We neglect here the tilt angles of the $\mathrm{C}-\mathrm{C}$ bonds.

Since the geometrical barrier monotonically increases with $n$ while $\zeta$ rapidly decreases, the length scale of injection exhibits a minimum that separates the two regimes of short and long chain lengths, as is shown in Figure 7.

At short chain length, the decrease of $\zeta$ dominates $\lambda$. This is due to the distance dependence of the energy mismatch between the Au Fermi level and the pentacene HOMO according to a power law:

$$
\Phi_{h}=-\frac{c}{\left[r_{\mathrm{Pen}}+r_{\mathrm{Au}-\mathrm{S}}+r_{\mathrm{S}-\mathrm{C}}+(n-1) l\right]^{m}}
$$

where the constant $\mathrm{c}$ and the exponent $m$ depend on the detailed electrostatic interaction (charge-induced dipole, induced dipoleinduced dipole, etc.), the orientation of the pentacene molecule with respect to the Au surface, and the integration of the molecule/surface interactions across the finite volume of the Au electrode. ${ }^{32}$

(28) Käfer, D.; Ruppel, L.; Witte, G. Phys. Rev. B: Condens. Matter Mater. Phys. 2007, 085309

(29) Bock, C.; Pham, D. V.; Kunze, U.; Käfer, D.; Witte, G.; Wöll, C. J. Appl. Phys. 2006, 114517

(30) Wang, S. D.; Dong, X.; Lee, C. S.; Lee, S. T. J. Phys. Chem. B 2005, 109, 9892-9896.

(31) Mayer, A. C.; Ruiz, R.; Headrick, R. L.; Kazimirov, A.; Malliaras, G. G. Organic Electronics 2004, 5, 257-263.

(32) Israelachvili, J. N. Intermolecular and Surface Forces, New York, Academic Press, 1985.

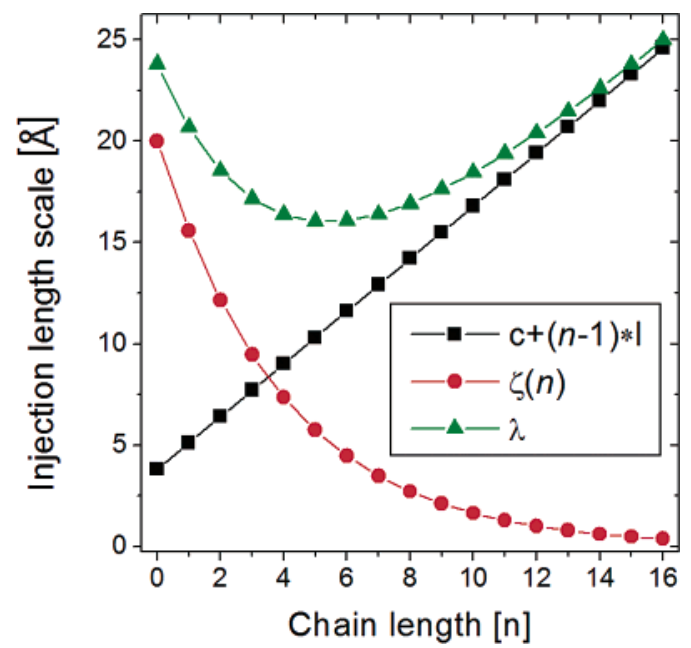

Figure 7. Terms contributing to the effective length scale for injection $\lambda$ (triangles). The values of the $c+(n-1) l$ term (squares) were taken from ref 16 , and the $\zeta(n)$ term is modeled by an exponential decay (circles).

The strength of the anisotropic pentacene/Au interaction will change abruptly because pentacene molecules grow on alkanethiols with their molecular long axis standing normal to the surface, whereas they are planarly oriented on bare $\mathrm{Au} .{ }^{26}$ The electrostatic interaction may extend deeper into the pentacene layer, because of the long-range character of eq 3 , and according to the electrical permittivity of pentacene. This contributes to create a finite length scale $\xi$ of energy disordered states.

The decrease of the voltage drop in the case of short-chain alkanethiols, $n<10$, depends on the effective coverage $\theta$ of the alkanethiol monolayer. Because short-chain alkanethiols do not yield compact SAMs,${ }^{33}$ the saturation current of short-chain alkanethiol FET can be regarded as the superposition of two independent contributions:

$$
\begin{aligned}
I_{\mathrm{sat}}=(1-\theta(n)) k(V g s- & \left.V r c_{\mathrm{s}}-\mathrm{V}_{\mathrm{T}}\right)^{2}+ \\
& \theta(n) k\left(\operatorname{Vgs}-\operatorname{Vrc}_{\mathrm{s}}(n, \Omega)-V_{\mathrm{T}}\right)^{2}
\end{aligned}
$$

where $\theta$ is the SAM coverage, $\Omega$ represents the angular coordinates of pentacene relative to the alkanethiol molecules, and the constant $k=1 / 2 W L^{-1} C_{\mathrm{i}} \mu$. Here $W$ is the channel width, $L$ is the length, and $C_{\mathrm{i}}$ is the capacitance of the gate dielectric. According to ref $16, \theta$ scales as $n^{2}$ until it reaches completion at $\theta=1$ for $n=10$. This square dependence versus $n$ does not explain our observation $\alpha=3-4$ in eq 1 and suggests that further interface effects have to be taken into consideration, as discussed in the next subsection.

When $n \geq 10$, the coverage $\theta$ becomes unit, the first contribution in eq 4 disappears, and the current is controlled by the SAM. Maximum current, and hence charge mobility, are in this case limited by the SAM interface resistance. This explains why mobility decreases exponentially versus $n$ for long chains.

This simple model accounts qualitatively for the basic features of the charge mobility and the potential profiles in Figure 1. However, it cannot explain the observation of the odd-even

(33) Camillone, N.; Leung, T. Y. B.; Schwartz, P.; Eisenberger, P.; Scoles, G. Langmuir 1996, 12, 2737-2746. 
effects. If we assume that the geometrical thickness of odd alkanethiols may be incrementally shorter than even ones, owing to the orientation of the last $\sigma$ bond that is tilted with respect to the $\mathrm{Au}$ surface, ${ }^{34}$ the odd terms would exhibit charge mobility larger than the even ones. Thus, the odd-even effect observed cannot be explained by simple geometrical arguments, and we need to consider in more detail the mechanism of charge injection across the alkanethiol layer using time-dependent perturbation theory.

III.2. Time-Dependent Perturbation Theory. The charge injection rate $\kappa$ between unperturbed pentacene states and $\mathrm{Au}$ can be expressed by means of time-dependent perturbation theory ${ }^{35}$ as

$$
\begin{aligned}
& \kappa=\kappa_{\text {direct }}+\kappa_{\text {interface }}+\kappa_{\text {through-bond }} \approx \kappa_{\text {direct }}+ \\
& \kappa_{\mathrm{Au}-\mathrm{Pen}} \kappa_{\mathrm{Pen}-\mathrm{Pen}, 0}+\kappa_{\mathrm{Au}-\mathrm{SAM}} \kappa_{\mathrm{SAM}} \kappa_{\mathrm{SAM}-\mathrm{Pen}, 0}+\ldots
\end{aligned}
$$

where $k_{\text {direct }}$ is the rate for direct (through-space) charge transfer between Au and "unperturbed" pentacene states in the channel, given by the Fermi golden rule:

$$
k_{\text {direct }}=\left|V_{\mathrm{Au}-\mathrm{Pen}, 0}(\lambda)\right|^{2} \delta\left(\omega_{\mathrm{Au}-\mathrm{Pen}, 0}\right)
$$

The matrix element $V_{\mathrm{Au}-\mathrm{Pen}, 0}$ is an exponentially decaying function of the charge injection $\lambda$, and Dirac's $\delta$ acts on the energy difference between Fermi level of $\mathrm{Au}$ and unperturbed HOMO of pentacene. This term increases as $\lambda$ is reduced.

The second term describes the injection from Au to pentacene in resonance with the $\mathrm{Au}$ Fermi level through energymismatched pentacene states:

$$
\begin{aligned}
& \kappa_{\mathrm{Au}-\mathrm{Pen}} \kappa_{\mathrm{Pen}-\mathrm{Pen}, 0}= \\
& \frac{\left|V_{\mathrm{Au}-\mathrm{Pen}}(\lambda-\zeta) V_{\mathrm{Pen}-\mathrm{Pen}, 0}(\xi)\right|^{2}}{\Phi_{\mathrm{h}}^{2}(n)} \delta\left(\omega_{\mathrm{Au}-\mathrm{Pen}, 0}\right)
\end{aligned}
$$

In eq 7 only one energy-mismatched pentacene state, whose energy shift corresponds to the hole injection barrier $\phi_{\mathrm{h}}$, is accounted for sake of clarity. For more than one energymismatched pentacene state, higher order terms should be introduced. The matrix element $V_{\mathrm{Au}-\mathrm{Pen}}$ decreases exponentially versus $n$, whereas $\Phi_{\mathrm{h}}$ decreases as a power law as in eq 3 . $V_{\text {Pen-Pen,0 }}$ will be a monotonically decreasing function of the length scale of energy disorder $\zeta$. At small $n$, the rate contribution eq 7 will grow as a power law with an exponent of order $2 m$ (see eq 4 ). This accounts for the trend observed at small $n$ in Figure 3, where exponents of 3.6-3.8 are measured. In the framework of eqs 5 and 7 , it turns out $m \approx 2$. This would be consistent with shift in the HOMO level of pentacene near the surface as due to charge-induced dipole interaction. At larger $n$, as $\zeta$ tends to zero this term converges to the direct transfer term in eq 4 , thus increasing the density of states.

The third contribution represents a charge-transfer pathway assisted by localized states along the alkanethiol molecule: ${ }^{17}$

(34) Rong, H.-T.; Frey, S.; Yang, Y.-J.; Zharnikov, M.; Buck, M.; Wühn, M.; Wöll, C.; Helmchen, G. Langmuir 2001, 17, 1582-1593.

(35) Cohen-Tannoudji, C.; Diu, B.; Laloë, F. Quantum Mechanics; Hermann: Paris, 1977; Vol. 2.

$$
\begin{aligned}
& \kappa_{\mathrm{Au}-\mathrm{SAM}^{-} \kappa_{\mathrm{SAM}} \kappa_{\mathrm{SAM}-\mathrm{Pen}, 0}=} \\
& \frac{\left|V_{\mathrm{Au}-\mathrm{S}}\right|^{2}}{\omega_{\mathrm{S}-\mathrm{E}_{\mathrm{F}}}^{2}} \frac{\left|V_{\mathrm{S}-\mathrm{C}}\right|^{2}}{\omega_{\mathrm{S}-\mathrm{E}_{\mathrm{F}}} \omega_{\mathrm{C}-\mathrm{E}_{\mathrm{F}}}} \frac{\left|V_{\mathrm{C}-\mathrm{C}}(l)\right|^{2(n-1)}}{\omega_{\mathrm{C}-\mathrm{E}_{\mathrm{F}}^{2(n-1)}}} \frac{\left|V_{\mathrm{C}-\mathrm{Pen}, 0}(\vartheta)\right|^{2}}{\omega_{\mathrm{C}-\mathrm{Pen}, 0}^{2}} \\
& \delta\left(\omega_{\mathrm{Au}-\mathrm{E}_{\mathrm{F}}}\right) \delta\left(\omega_{\mathrm{E}_{\mathrm{F}}-\mathrm{Pen}, 0}\right)
\end{aligned}
$$

In eq 8 it is assumed that no disorder is present at the interface between pentacene and SAM. Since $\mathrm{V}_{\mathrm{C}-\mathrm{C}} \approx \exp \left(-1 /{ }_{2} \beta l\right)$, then the rate will decrease exponentially versus $n$ with the inverse decay length $\beta$. The term $V_{\mathrm{C}-\mathrm{Pen}, 0}$ is sensitive to the relative orientation $\vartheta$ of the pentacene molecules with respect to the terminal $\sigma$ bond, for example, via $\cos ^{2 j} \vartheta, j$ being an integer number. This term discriminates between the even and odd terms since (i) pentacene molecules grow on alkanethiols approximately normal to the Au surface ${ }^{26}$ and (ii) the terminal $\sigma$ bond of alkane thiols points normal to the surface for even terms and is tilted for odd terms. ${ }^{33}$ Equation 8 explains the odd-even effect in the charge mobility and the experimental value of $\beta$ in the long-chain regime which is twice the decay rate of the matrix element $V_{\mathrm{c}-\mathrm{c}}$.

With no alkanethiol layer, only eqs 5 and 6 contribute to the current. In the short-chain regime, there is entanglement between geometrical, electronic, and morphological effects. The monolayer formed by short-chain alkanethiols is not compact ${ }^{33}$ as the alkanethiol molecules undergo substantial lateral diffusion. The increasing trend in the mobility reflects the coverage of the electrode, ${ }^{33}$ and hence the improvement of the SAM/ pentacene interface, as well as the decrease of the hole injection barrier. To these, a smaller thickness of the short-chain alkanethiol layer with respect to the stretched (all-trans) chain may add. ${ }^{16}$

At the crossover from short- to long-chain, the thickness of the alkanethiol barrier undergoes a transition toward the alltrans conformation around $n=8,{ }^{16}$ a compact SAM is formed and pentacene grows in a $2 \mathrm{D}$ mode with molecules normal to the SAM surface. The maximum in the charge mobility arises because of the compromise between the smaller hole injection barrier, the optimum morphology, and the minimum thickness of the alkanethiol layer. The effect of the increasing barrier thickness starts to dominate as the HOMO levels in the molecules at the interface become quasi-resonant with Au Fermi level and there is no longer a benefit from the decrease of the hole barrier.

III.3. The Organic FET as a Charge Injection Device. In the long-chain regime, the charge injection through the thick barrier controls the response of the device. The inverse decay length of the charge mobility $\beta=0.5-0.6 \AA^{-1}$ lies in the range $0.5-1 \AA^{-1}$ reported for the exponential decay of the current flowing across mesoscopic metal-alkanethiol-metal junctions, ${ }^{2,36}$ as well as the value of 0.6 reported for metalalkanedithiol-conductive polymer-metal junctions. ${ }^{8}$ In earlier experiments, a through-bond charge-transfer mechanism following the $\sigma$-orbital pathway was invoked in order to explain the slow decay rate $\beta$.

From section III.2, it turns out that the odd-even fluctuations in the charge mobility are consistent with the charge injection mechanism assisted by alkanethiols. We neglect the influence on the morphology because the morphological data do not

(36) Yamamoto, H.; Waldeck, D. H. J. Phys. Chem. B 2002, 106, 7469-7473. 
exhibit odd-even effect, and, above all, because the odd-even fluctuations remain of the same relative intensity (Figure 3) also when the morphology of the pentacene film is "invariant" versus $n$. We have not yet structural support from diffraction experiments to rule out a modulation of the structure of the pentacene/ SAM interface versus $n$. This assessment requires further investigation by structural techniques.

III.4. The Organic FET as a Sensitive Gauge to Submolecular Length Scale Details. In the hypothesis that the oddeven fluctuations arise solely from the electronic properties of the alkanethiols, we identify two possible origins: (i) the modulation of the overlap between the terminal group of the alkanethiols and the pentacene molecules, as discussed with eq 8 , and (ii) the modulation of the surface dipole and hence the energy level of the alkanethiol resonance coupled to Au. ${ }^{37}$ This would enter in eqs 3 and 7.

We cannot discriminate experimentally the dominant effect, but it is possible to make an educated guess. In the literature of SAMs, odd-even effects in the XPS spectra have been ascribed to the interactions of sulfur with different $\mathrm{Au}$ sites in the case of aromatic SAMs. ${ }^{38}$ In the case of alkanethiols, the modulation of the dipole should affect mainly the $\sigma$ bond $^{2,36,37}$ rather than the $\mathrm{Au}-\mathrm{S}$ resonance located $1.4 \mathrm{eV}$ below the Au Fermi level. ${ }^{37}$ In any case, since the alkyl chain is weakly polarizable, the fluctuations of the surface dipole of alkanethiols, must be very small, ${ }^{39}$ consistent with the conclusions of ref 37 . Moreover, to be consistent with our data, the dipole of the odd alkanethiols should be larger, which is at variance with the trend of interfacial energy reported in ref 40 .

We conclude that the overlap between the $\sigma$ bond and the pentacene owing to the orientation of the terminal bond of the alkyl chain appears as a more plausible origin at this stage. This orientation-dependent coupling between a conjugated moiety and an alkyl chain has been invoked to explain odd-even fluctuations in photoinduced molecular charge transfer between donor-acceptor pairs spaced by an aliphatic chain. ${ }^{41}$ In the super-exchange framework, ${ }^{36}$ the terminal $\mathrm{C}-\mathrm{C}$ bond in the alkyl chain couples directly to the hole-donor state, namely, the HOMO $\pi$ orbital in pentacene, as in eq 8 . In even alkanethiol chains, this bond points normal to the Au surface, for the odd chains, it is tilted by $\vartheta=71^{\circ}$ with respect to the surface normal. ${ }^{42}$ Since pentacene is normal to the SAM, ${ }^{26}$ then the symmetry of the wave function makes roughly the overlap between the HOMO of pentacene and that of the even-term alkanethiol larger at least by a factor $1 / \cos ^{2} \vartheta \approx 10$. This is a maximum enhancement in the hypothesis that each chain is coupled to a pentacene molecule. Fluctuations between adjacent odd-even terms as from Figure 3 are of the order of $3-5$ times.

To compare with the earlier current versus $n$ experiments where no odd-even effect was ever observed, we can say that

(37) Alloway, D. M.; Hofmann, M.; Smith, D. L.; Gruhn, N. E.; Graham, A. L.; Colorado, R.; Wysocki, V. H.; Lee, T. R.; Lee, P. A.; Armstrong, N. R. J. Phys. Chem. B 2003, 107, 11690-11699.

(38) Heister, K.; Rong, H. T.; Buck, M.; Zharnikov, M.; Grunze, M.; Johansson, L. S. O. J. Phys. Chem. B 2001, 105, 6888-6894.

(39) Odd-even fluctuations of the surface dipole have been reported in fluorinated SAMs only. ${ }^{37}$

(40) Bain, C. D.; Troughton, E. B.; Tao, Y. T.; Evall, J.; Whitesides, G. M.; Nuzzo, R. G. J. Am. Chem. Soc. 1989, 111, 321-325.

(41) Ryu, C. K.; Wang, R. Y.; Schmehl, R. H.; Ferrere, S.; Ludwikow, M.; Merkert, J. W.; Headford, C. E. L.; Elliot, C. M. J. Am. Chem. Soc. 1992 114, 430-438.

(42) Angelico, V. J.; Mitchell, S. A.; Wysocki, V. H. Anal. Chem. 2000, 72, 2603-2608 the odd-even effects in the present experiment emerge because the pentacene molecules act as probes with unique features: well-ordered alkanethiol-pentacene interface with a precise orientation between alkanethiol and pentacene molecules and sharpness in energy and anisotropy of the pentacene relevant HOMO state. The lack of earlier observations can be ascribed to the different configuration used, namely, a conductive probe (nano- or microscopic) on top of the SAM/Au interface. All probe electrodes used were isotropic in their structure so the odd-even effects are averaged out by the configuration of the $\mathrm{SAM} /$ probe interface.

It is evident that the odd-even fluctuations, independently of the detailed origin, demonstrate that the transistor response through charge injection is extremely sensitive to changes in submolecular features at the interface. This prompts an interesting proposition in terms of using a pentacene FET, or a FET with another suitable organic semiconductor, as an experimental gauge to measure the charge transport across a monolayer adsorbed on the source-drain electrodes. This configuration has several advantages with respect to the configurations based on two electrodes: ${ }^{17}$ (i) the interface is geometrically well defined; (ii) the probe (pentacene) is sharp in energy and is deposited by clean, low-energy techniques; (iii) the sign and density of free carriers involved in the charge injection throughout can be selected with the gate voltage. Our data, indeed, show unambiguously that holes are transported across the alkanethiol monolayers.

\section{Conclusions}

Our work demonstrates major effects of the organic/metal interface in organic transistors: (i) the interfacial barrier between the gold electrodes and pentacene largely controls the transistor response; (ii) the length scale of charge injection is determined by the interplay between ordering, morphology and electronic properties of the injection interface; (iii) the enhancement of the charge mobility occurs as the length scale of charge injection decreases; (iv) as the alignment between metal and organic semiconductor energy levels is achieved and the morphology of the interface are invariant, the charge mobility is solely controlled by the charge injection across a single monolayer junction. For the first time, odd-even effects are reported in a charge transport experiment across alkanethiol monolayers, because the anisotropic probe (pentacene) is ordered with respect to the alkanethiol monolayer, and the pentacene states coupled to the alkanethiol are narrowly distributed in energy. These results suggest that a FET with a suitable organic semiconductor as a probe can be used as a sensitive and controlled gauge for studying charge transport across homologue series of selfassembly monolayers.

Acknowledgment. We are grateful to Jérôme Cornil for sharing preliminary data on charge-transfer model calculations. We also thank D. D. Sarma, G. Scoles, D. Cahen, P. Heremans, and one of the referees for helpful and constructive discussions. This work is supported by EU-NMP Integrated Project 500345 NAIMO. P.S. and R.K. acknowledge ICTP for TRIL Fellowship.

Supporting Information Available: Information about electrical measurements done on FET. This material is available free of charge via the Internet at http://pubs.acs.org.

JA069235M

6484 J. AM. CHEM. SOC. - VOL. 129, NO. 20, 2007 